\title{
Characterization of a carbon felt electrode: structural and physical properties
}

\section{J. González-García ${ }^{\mathrm{a}}$, P. Bonete ${ }^{\mathrm{a}}$, E. Expósito ${ }^{\mathrm{a}}$, V. Montiel*a ${ }^{* a}$ A. Aldaz and R. Torregrosa-Maciáb}

a Grupo de Electroquímica Aplicada. Departamento de Química Física. Universidad de Alicante. Apartado de Correos 99. 03080 - Alicante. SPAIN.

b Departamento de Química Inorgánica. Universidad de Alicante.

* Author to whom correspondence should be addressed

The aim of this paper is the characterization of a carbon felt (Le Carbone Lorraine, RVC 4002) to be used as three-dimensional electrode. A wide group of very different techniques were used in the physical and structural characterization of this material. Both, structural (porosity, mean pore radius, specific surface area, tortuosity) and physical properties (permeability, electrical resistance) were determined by using mercury porosimetry, adsorption isotherm analysis, a filamentary analog procedure and liquid permeametry (pressure drop method). The results obtained from the modelling of the hydrodynamic behaviour, from previous work, were also applied here. The carbon felt studied was found to have a porosity around 0.98 , a specific surface area of $22100-22700 \mathrm{~m}^{-1}, 2.710^{-3} \Omega \mathrm{m}$ electrical resistivity and a tortuosity between 5 and 6 . The comparison of these results with those found in the literature for other similar materials, used as three-dimensional electrodes, highlights the attractions of this 
carbon felt as a three-dimensional electrode.

The increasing use of electrochemical technologies in a variety of applications such as synthesis, energy storage and environmental treatment, is due, among other reasons, to the utilization of porous materials as threedimensional electrodes in the design of electrochemical reactors. One of the main advantages of this kind of electrodes has derives from the fact that it can provide a more compact reactor for a given duty, together with a high fractional conversion per pass ${ }^{1}$. From the kinetic point of (and in many cases from the beginning of the process), the process will become controlled by the convection-diffusion mass transport from the bulk solution to the electrode surface. Under these conditions, the mathematical expression for the process current is given by the limiting current (1).

$$
I_{L}(t)=z F k_{m} \operatorname{Ac}(t)
$$

For a given fluid, the limiting current value depends on two designable parameters, viz. the mass transport coefficient, $k_{m}$, and the electrode area, $A$. Therefore, the limiting current can be increased by (i) improving internal agitation inside the reactor (by changing the reactor design, in a suitable way, in order to increase $k_{m}$ ) or (ii) increasing the electrode area, by incorporating a three-dimensional electrode into the reactor. For this second 
option, equation (1) is transformed in (2)

$$
\mathrm{I}_{\mathrm{L}}(\mathrm{t})=\mathrm{zFk}_{\mathrm{m}} \mathrm{A}_{\mathrm{e}} \mathrm{V}_{\mathrm{e}} \mathrm{c}(\mathrm{t})
$$

$A_{e}$ is the specific surface area of the porous material $\left(\mathrm{m}^{2} / \mathrm{m}^{3}\right.$, surface/ volume relationship for the electrode) and $V_{e}$ is the electrode volume ${ }^{2}$.

It is important to characterize the intrinsic properties of the porous material when designing electrochemical reactors with three-dimensional electrodes by determining, not only the structural properties (such as pore size distribution, specific surface area, porosity, isotropy, tortuosity...), but also its physical properties (such as hydraulic diameter, electrical conductivity, permeability...).

A survey of the literature shows a large number of papers dealing with the characterization of several materials used as three-dimensional electrodes, particularly metallic foams ${ }^{3-7}$. However, we have found few papers dealing with the study of the properties of carbon felts, in spite of its attractive characteristics and applications $^{8-11}$ (i.e. electrosynthesis ${ }^{12}$, metal recovery ${ }^{13}$ ), probably due to the fact that the hydrophobic nature and the compressibility of this material restrict its applications.

The influence of the (felt/reactor compartment) thickness ratio on the hydrodynamic behaviour of a filter-press electrochemical reactor was studied in a previous paper ${ }^{14}$. To complete this previous study, the values 
obtained in the characterization of a carbon felt (RVC 4002 supplied by Le Carbone Lorraine) in this paper will be compared with those, provided in the literature, for similar carbon felts and other materials used as threedimensional electrodes.

\section{Experimental Details}

\section{Porous material}

In this study, the RVC 4002 Carbon felt (0.01 m thickness, $100 \mathrm{~kg} \mathrm{~m}^{-3}$ of density, $96 \%$ carbon content, $1100 \mathrm{~m}^{2} \mathrm{~kg}^{-1}$ of specific surface area $\left(\mathrm{N}_{2}\right)$ and electrical resistivity in the long direction of $0.2 \Omega \mathrm{m}$ and in the short one of $0.02 \Omega \mathrm{m}$,as supplied by the Le Carbone Lorraine) was used.

The material is supplied in sheets of $10 \mathrm{~mm}$ thickness. A first visual inspection shows the sheets formed by other thinner sheets binded between them, so that a different texture is presented as a function of the side observed. The thickness of the three-dimensional electrode $(8,9,10$ and 13 $\mathrm{mm}$ ) can be achieved by adding sheets or a thinner sheet obtained by cutting an original sheet.

\section{Apparatus}

The scanning electron microscope (SEM) images were obtained in a JSM840 JEOL microscope.

Pressure drop measurements through the carbon felt were carried out using 
the experimental set up in the Figure $1^{14}$, with a filter-press electrochemical reactor as a test cell $(0.008 \mathrm{~m}$, compartment thickness). Taking into account the highly hydrophobic behaviour of the carbon felt, the specimens were immersed in an ultrasonic bath with water for 2 hours to ensure the complete de-aeration and effective wetting before use. In order to avoid gas occlusion into the porous medium, water was also de-aerated before each experiment by $\mathrm{N}_{2}$ bubbling.

Mercury porosimetry was carried out in a mercury porosimeter CARLO ERBA model 2000.

The helium density (true density) at room temperature was determined in an automatic adsorption apparatus (OMNISORP-100). This same machine was used to obtain the nitrogen adsorption isotherms at $77 \mathrm{~K}$ which were analyzed by the BET method ${ }^{15}$ to obtain the porosity parameters of the carbon felt.

The true density was also determined in a METTLER TOLEDO AG204 (analytic scale) equipped with a density determination device.

\section{Experimental procedure}

Drop Pressure measurements. These were carried out with the experimental arrangement shown in Figure 1, equipped in each case with different type of pressure gauges. The liquid (water in this case) was fed into the reactor by a centrifuge pump model MSE-EP-R (March May). 
Variable area flow-meters and polypropylene membrane valves were used to control the liquid flow. All-glass heat exchangers and Pt-100 probes were used to control the temperature during the drop pressure measurements.

Electrical resistivity measurements. The apparent electrical resistivity is obtained from the slope of the experimental polarization curves. These plots were recorded for two perpendicular directions employing air injection at $298 \mathrm{~K}$. For one, the current passes the length way (from 10 to $35 \mathrm{~cm}$ distance between the measurement points), and current values ranging from 0.1 to 1.3 A were used to determine the voltage. And for the other direction, thickness way $(1 \mathrm{~cm})$, lower current values than former were used $(\mathrm{I}<0.25$ A). We found contact problems, and some other experimental difficulties, when measuring the electrical resistivity through this short direction, generally due to the small dimensions of the material.

\section{Results}

\section{Structural properties}

Structure and fibre size (SEM). The corresponding micrographs of the carbon felt, Figure 2, show an anisotropic medium constituted by randomly dispersed fibres. The void space between fibres is large (so that a high value of porosity can be expected) and homogeneously distributed, with a 
geometrical shape quite different from other materials currently used as three-dimensional electrodes. Because of these characteristics, some properties, such as pore diameter have no meaning from a geometrical point of view. The single fibres observed have a flat, cylinder-like shape, with shallow grooves along the long axis; a more detailed inspection reveals each fibre is formed by a group of thinner fibres, melted together lengthways. A value for the fibre diameter of $d_{f}=(19.0 \pm 0.2) 10^{-6} \mathrm{~m}$, was obtained from the average dimensions taken from micrographs.

Porosity, $\varepsilon$. Porosity was determined by using different methods like density and modelling of Residence Time Distribution (RTD) methods.

Helium density and mercury porosimetry (density methods). The apparent density of the felt, $\rho_{A}$, was determined with a dilatometer prepared for mercury porosimetry, placed in the mercury filling unit, by using a slight modification of the currently used procedure. Previously outgassed (at room temperature and $10^{-3}$ torr), the dilatometer, containing the specimen, was filled under vacuum with mercury up to the calibrated volume level. The pressure was then allowed to increase, under control, up to atmospheric pressure, giving a macropore volume $\left(\mathrm{r}_{\mathrm{p}}<25 \mathrm{~nm}\right)$ of $1.8 \mathrm{~cm}^{3} \mathrm{~g}^{-1}$ corresponding to a macropore surface of $0.19 \mathrm{~m}^{2} \mathrm{~g}^{-1}$. After that, the dilatometer, with felt and not refilled with mercury, was reweighed in order to determine the density of the uncompressed felt 
obtaining a value of the apparent density of $0.351 \mathrm{~g} \mathrm{~cm}^{-3}$.

The refilling with mercury up to the calibrated volume level, at atmospheric pressure, will give rise to a density higher than that of the uncompressed material, because the carbon felt has a large volume of macropores, and it is also compressible. The refilling with mercury, at atmospheric pressure, causes the decrease in void volume, not only by filling the large macropore volume with mercury, but also by decreasing the size of the specimen by compression, as a consequence of the mercury column weight. In this case, the application of the conventional determination procedure results in mercury occupying the porosity of larger size, giving a value of apparent density of $0.95 \mathrm{~g} \mathrm{~cm}^{-3}$, higher than that obtained for the uncompressed felt. The determination of the volume of lower size porosity was carried out in the mercury porosimeter after refilling with mercury. The pore volume obtained for this range of pore size $\left(3.5<\mathrm{r}_{\mathrm{p}}<15 \mathrm{~nm}\right)$ was $0.7 \mathrm{~cm}^{3} \mathrm{~g}^{-1}$ corresponding to a total area of $176 \mathrm{~m}^{2} \mathrm{~g}^{-1}$. Figure 3 shows the size distribution curve for this porosity range. There are two well-defined pore ranges, mesopores between $3.5-20 \mathrm{~nm}\left(3.5-20 \times 10^{-3} \mu \mathrm{m}\right)$ and macropores with pore radius higher than $5000 \mathrm{~nm}(5 \mu \mathrm{m})$.

The true density of the sample, $\rho_{\mathrm{R}}$, was determined by using helium, a monoatomic molecule, small enough to be accessible to the lowest size micropores $(\cong 2 \AA)$ in the material. The obtained value was $2.17 \mathrm{~g} \mathrm{~cm}^{-3}$, which is close to that of graphite $\left(2.25 \mathrm{~g} \mathrm{~cm}^{-3}\right)$. This fact shows that carbon 
is the main component of the material (according to the $96 \%$ carbon composition, supplied by the manufacturer) and there is no helium adsorption.

Porosity, $\varepsilon$, can be calculated from the values of apparent and true densities, according to equation (3)

$$
\varepsilon=\frac{\left(\rho_{\mathrm{R}}-\rho_{\mathrm{A}}\right)}{\rho_{\mathrm{R}}}
$$

Giving a value of 0.84 .

Modelling of RTD. The carbon felt presents an uncommon property compared to other materials used as three-dimensional electrodes, its compressibility. Because of this, it is possible the addition of carbon felt sheets, up to obtain a geometrical volume higher than that of the compartment in the electrochemical reactor, without any physical damage resulting for the felt. This fact provides several advantages for its application, i.e. giving rise to a good electrical connection between the collector and the felt. Besides, despite of the hydrophobic nature of the carbon felt, its use provides a more efficient percolation of the fluid, without by-pass channelling between walls and porous material. These aspects were discussed in the previous paper ${ }^{14}$, analyzing the experimental residence time distribution, RTD, for different thickness ratios (felt/compartment) by 
simulating with models. One of the parameters optimized with these models was the porosity of the medium, $\varepsilon$. The results obtained for different thickness ratios were $0.984(8 / 8), 0.980(9 / 8) 0.976(10 / 8)$ and $0.970(13 / 8)$.

Specific surface area, $\mathbf{A}_{\mathbf{e}}\left(\mathrm{m}^{2} \mathbf{m}^{-3}\right)$. The value of specific surface area of a porous material seems to be dependent on the method used ${ }^{16}$ for its determination. We can find very different methods in the literature, such as (i) structural methods: the filamentary analog procedure ${ }^{9}$, (ii) physical methods: physical adsorption of gases $^{5}$, permeametry ${ }^{7}$, mercury porosimetry $^{17}$ and (iii) electrochemical mehods ${ }^{18}$. These provide a wide range of results and thus the evaluation of the true interfacial area, available for the reaction, is uncertain. The choice of a suitable method is conditioned by the nature of the specific surface area to be quantified and, because of that, the more suitable strategy will be to perform a comparative study of several techniques.

Filamentary analog. This method considers fibres as long, smooth, perfect non-porous cylinders. The specific surface area is related to the diameter of the fibre, $d_{f}$, and to the porosity of the material, $\varepsilon$, following equation (4). Nevertheless, examination under the SEM revealed some external roughness on fibres so that the validity of the specific surface area determined by this method is far from proven.

$$
A_{e}=\frac{4}{d_{f}}(1-\varepsilon)
$$


By using this equation with both porosity values obtained experimentally (from density and modelling methods), the specific surface area values deduced differ by a factor of 10 . With the porosity value from the density method, the specific surface area gives rise to a value of $33684 \mathrm{~m}^{2} \mathrm{~m}^{-3}$, in contrast with $2269 \mathrm{~m}^{2} \mathrm{~m}^{-3}$, obtained by using the porosity value from modelization $^{14}$.

Pressure drop method (permeametry). The pressure drop, as a function of the linear behaviour of the fluid velocity, has been analyzed by the Ergun equation, which describes the dissipation of mechanical energy in the flow through a porous medium ${ }^{19}$ :

$$
\frac{\Delta \mathrm{P}}{\mathrm{L}}=\mathrm{Mv}^{2}+\mathrm{Nv}
$$

Where $L$ is the porous material length in the flow direction and $v$ is the linear velocity of the fluid when the test cell is empty. The first term on the right side of the equation 5 , proportional to the square velocity, represents the contribution from turbulence flow and is due to inertial forces and direction changes of the fluid streams. The second term, proportional to the fluid velocity, represents the contributions from laminar flow and it is related to the viscous resistance at the porous material. Several flow regimes in porous media are proposed in the literature ${ }^{20}$ these regimes being 
characterized by the interstitial Reynolds number $\mathrm{Re}_{\mathrm{i}}$

$$
\operatorname{Re}_{i}=\frac{\rho \mathrm{vd}_{\mathrm{p}}}{\mu \varepsilon}
$$

$d_{p}$ is the pore equivalent diameter and $\rho$ and $\mu$ are the fluid density and the dynamic viscosity of the fluid, respectively. The four regimes are:

(i) Darcy or creeping flow regime, $\mathrm{Re}_{\mathrm{i}}<1$, (when viscous forces dominate).

(ii) Inertial flow regime, showing a non-linear relationship between pressure drop and flow rate. From $\mathrm{Re}_{i}=1-10$ to 150 , (steady non-linear laminar flow characterized by more pronounced boundary layers and early inertial nuclei).

(iii) Unsteady flow regime, $\mathrm{Re}_{\mathrm{i}}$ between $150-300$.

(iv) Chaotic regime, $\mathrm{Re}_{\mathrm{i}}>300$.

In our experimental conditions, with carbon felt $\left(\mathrm{Re}_{\mathrm{i}}<15\right)$, creeping flow and inertial flow regimes dominate. The empirical constants $M$ and $N$, depending on geometrical and physical parameters of the system, can be correlated to a specific surface area per volume unit of solid, $A_{s}$ [related to $A_{e}$ through $\left.\mathrm{A}_{\mathrm{e}}=\mathrm{A}_{\mathrm{s}}(1-\varepsilon)\right]$ and to the mean tortuosity, $T$, by means of the following expresions ${ }^{21}$ : 


$$
\begin{aligned}
& A_{s}^{4}=\frac{N^{3}(0.096 L \rho)^{2}}{M^{2}(5 \gamma \mu L)^{3}} \frac{\varepsilon^{3}}{(1-\varepsilon)^{4}} \\
& T^{4}=\frac{M^{2} 5 \gamma \mu L}{N(0.096 \rho L)^{2}} \varepsilon^{3}
\end{aligned}
$$

$\gamma$ is the circularity factor of the pores. A value between 1 and 1.5 times of some geometric sections of the pore is suggested in the literature. Here, a 1.25 factor has been applied because it has no great influence on the final result.

Figure 4 shows the $\Delta P / L v$ versus $v$ plot for different thickness ratios. The specific surface area resulting from the application of the Ergun method, by using the porosity values obtained from simulation for the different thickness ratios were 13300 (8/8), 15900 (9/8), 22700 (10/8) and 22100 $(13 / 8)$. It is to be notice that, for ratios up to $10 / 8$, the higher the thickness ratio, the greater the value of the specific area is, trending to reach a constant value when going from 10/8 to $13 / 8$ ratios.

Mercury porosimetry. Mercury porosimetry gives a macropore surface value of $0.19 \mathrm{~m}^{2} \mathrm{~g}^{-1}$. Taking into account the apparent density of $0.351 \mathrm{~g}$ $\mathrm{cm}^{-3}$, also determined by this method, a specific surface area of $6.65 \times 10^{4}$ $\mathrm{m}^{2} \mathrm{~m}^{-3}$ is obtained. On the other hand, a calculated area for mesopores of $176 \mathrm{~m}^{2} \mathrm{~g}^{-1}$ gives rise to a specific surface area of $6.18 \times 10^{7} \mathrm{~m}^{2} \mathrm{~m}^{-3}$ that, 
obviously, is excessively high.

Adsorption isotherms. Physical gas adsorption isotherm is a common method used to determine the specific area of porous solids. The apparent surface area of carbon was obtained from $\mathrm{N}_{2} / 77 \mathrm{~K}$ adsorption isotherms by applying the BET method ${ }^{15}$. Figure 5 shows the $\mathrm{N}_{2} / 77 \mathrm{~K}$ adsorption isotherm of carbon felt. This isotherm corresponds to the type II of the Brunauer, Deming, Deming and Teller (BDDT) classification ${ }^{22}$, typical of non-porous solids where a weak gas-adsorbent interaction is present. Because of the practically absence of microporosity, the isotherm obtained is linear. From this, a volume of the monolayer of $\mathrm{N}_{2}$ adsorbed of $1.84 \mathrm{~cm}^{3}$ $\mathrm{g}^{-1}$ is obtained, corresponding to an apparent surface area of $8 \mathrm{~m}^{2} \mathrm{~g}^{-1}$, which means a specific surface area of $8 \times 10^{5} \mathrm{~m}^{2} \mathrm{~m}^{-3}$.

Tortuosity. The tortuosity values for carbon felt obtained by using Ergun method were $4.95(8 / 8), 6.35(9 / 8), 5.07(10 / 8)$ and 6.04 (13/8). Tortuosity is a measure of the fluid path length through the porous material related to the actual path length through the carbon felt $^{23}$. From the experimental values observed, 5-6, there is no clear dependence on the compression the carbon felt was submitted.

Average pore diameter. It is evident that the concept of average pore diameter for a porous material, constituted by a framework of fibres, has no 
physical meaning. Nevertheless, the estimation of this allow us to establish the value of certain useful parameters (i.e. $\mathrm{Re}_{\mathrm{i}}$ ) for this comparative study. The average pore diameter can be obtained from the results of the Ergun equation or by the filamentary analog method. The average pore diameters obtained for the different thickness ratios used were (3-2) $\times 10^{-4} \mathrm{~m}$ from Ergun method and $1 \times 10^{-3}(8 / 8), 9 \times 10^{-4}(9 / 8), 8 \times 10^{-4}(10 / 8)$ and $6 \times 10^{-4}$ m (13/8) from filamentary analog method. These values obtained from the filamentary analog method are higher than fibre gap in one order of magnitude, as it can be appreciate in SEM micrographs, for a thickness ratio of $8 / 8$.

\section{Physical properties}

Permeability. Permeability, $\mu / \mathrm{N}$, defined based on the Darcy law, is the velocity of a fluid, through a volume unit of a porous medium, per unit of differential pressure. As long as it is exclusively dependent on the porous media properties, the value of the permeability coefficient is frequently used to give an indication of the ease of passing a fluid through a porous material. It can be found in the literature ${ }^{24}$ some values of permeability coefficient for different particulate beds, showing that permeability coefficient can vary over a wide range of values. From those values one can deduced that the higher the porosity, the easier the fluid flows through a porous material, so that the permeability coefficient will increase with porosity. 
The permeability values for carbon felt obtained by using Ergun method were $2.93 \times 10^{-10}(8 / 8), 1.23 \times 10^{-10}(9 / 8), 9.35 \times 10^{-11}(10 / 8)$ and $6.90 \times 10^{-11} \mathrm{~m}^{2}(13 / 8)$. As it could be expect, the permeability coefficient values, found in this work for the carbon felt, increase as thickness ratio decreases or, in other words, when the felt compression decreases. Nevertheless, they are lower than that found in the literature ${ }^{24}$, in spite of the porosity is very high in this case. Moreover, the highly hydrophobic nature of carbon materials, which differs from that of the other materials used in the literature, could account for this behaviour.

Electrical resistivity. The polarization curves through the large direction were highly reproducible for different distances between probes. The electrical resistivity values obtained were $2.67 \times 10^{-3}, 2.71 \times 10^{-3}$ and $2.70 \mathrm{x}$ $10^{-3}$ giving a mean value of $2.7 \times 10^{-3} \Omega \mathrm{m}$, quite different from those supplied by the manufacturers.

The values obtained $\left(2.4 \times 10^{-1} \Omega \mathrm{m}\right)$ thickness way, were quite non reproducible and they differ from that obtained length way, and those, from the manufacturer. It is to be noticed that both values obtained here are quite different from the electrical resistivity of another porous materials found in the literature ${ }^{5}$. This reveals the high isotropy of the carbon felt, higher than that observed for another porous material such as Ni foam.

The electrical resistivity, $\mathrm{r}_{\text {felt }}$, of a porous material can also be calculated 
from equation (9) 5

$$
\mathrm{r}_{\mathrm{felt}} \approx \frac{4}{(1-\varepsilon)} \mathrm{r}_{\mathrm{C}}
$$

$r_{C}$ is the electrical resistivity of the own material the electrode is made of. Because of the great number of different carbon types, it can be found in the literature a wide range of electrical resistivity values for carbon, but all of them fall between $4 \times 10^{-5}$ and $6.5 \times 10^{-5} \Omega \mathrm{m}$. In this work, we have obtained $\mathrm{r}_{\text {felt }}$ values ranging from $1 \times 10^{-2}$ to $1.63 \times 10^{-2} \Omega$ m by using the $\mathrm{r}_{\mathrm{C}}$ values found in the literature in equation $6(\varepsilon=0.984$, calculated by simulation for the $8 / 8$ thickness ratio). From this, it is deduced that experimental values found for both current pathways are in the theoretical range.

\section{Discussion}

Table 1 summarize the results obtained in this work together with other data from the literature, in order to discuss the next properties of the materials used as three-dimensional electrodes.

\section{Porosity, $\varepsilon$}

Nearly all researchers use to estimate the porosity of the three-dimensional electrode from the material $\left(r_{c}\right)$ and porous medium $\left(r_{\text {felt }}\right.$, in this case) 
density throughout the geometrical volume of the specimen. This method gives good values for Ni foam but, since it is difficult to determine the true dimensions of a compressible specimen, it would seem quite inadequate its application to carbon felts. Another authors prefer to use apparent (mercury) and true (helium) density for porosity calculations. In this method, both the true dimensions and the fibrous structure of the felt will be modified -by the static pressure of mercury and by the mercury intruded-, giving rise to a unrealistic values for the apparent density. Neither the former nor this last one seems to be quite suitable for compressible materials such as carbon felt. Taken this in mind, it is easily understandable the great dispersion of porosity values found in the literature. Moreover, carbon felt shows a filamentary microscopic structure, of approximately 20 $\mu \mathrm{m}$ of fibre diameter, and it would be unreasonable it had a porosity lower than $\mathrm{Ni}$ foam having a bigger skeleton dimensions.

In contrast with the results reported by using those methods, simulation yielded porosity values higher than 0.97 . The most noticeable feature of this is that, being a hydrodynamic method for the determination of the porosity of a porous medium, porosity values are obtained in situ. That is, in a similar situation to where electrode will be used in a practical application. Moreover, simulation method yields the porosity of the porous medium as a function of the thickness ratio, which makes these results very suitable to be used in the design of some aspects of the reactors in 
electrochemical engineering applications.

\section{Specific surface area, $A_{e}\left(m^{2} \mathbf{m}^{-3}\right)$}

Specific surface area data for some porous materials, used as threedimensional electrodes, in the literature and these obtained in this work are summarized in Table 1. Specific surface area values found in the literature have been obtained almost exclusively by using the filamentary analog for carbon felts, although this method involves inadequate approximations. From SEM micrographs, not always the fibres constituting the carbon felt can be considered like cylinders with a smooth surface and having no surface porosity, as it happens in this particular case. Moreover, the value obtained for the specific surface area depends on the porosity, being this difficult to determine because of the compressibility of the porous medium. In spite of this, we have calculated the specific surface area by using the filamentary analog for two porosity values obtaining quite different results. We have also used mercury porosimetry and gas adsorption isotherms to determine the specific surface area, regardless of the reasons for which the results of these methods are unsuitable for their use. With compressible materials, the first provides unrealistic results as we have comment in the porosity discussion. And the results of the second are not suitable in electrochemical applications for the next reasons.

Isotherm adsorption techniques provide specific surface area results by 
mathematics treatment of the experimental data by calculating pore volumes. The assessment of porosity of solid needs to use different techniques depending on the pore size range (based each one on a different theoretical approach). Besides, the fluid used (gas, in the isotherm experiments or liquid in the adsorption from solution isotherms) to determinate the porosity depends on the pore size range covered by the fluid. In this way, the interaction nature (solid/liquid) or (solid/gas) will determine a different extension of pore ranges, because of this the specific surface area values will correspond to different sample domains.

Attending to the interaction type in electrochemical processes and in isotherm adsorption from solution, being equal, this last one will be the more suitable method to determine the true specific surface area; but this kind of technique requires equilibrium times very large that make its use very unoperative. Because of this we decided to use the variation in the mercury porosimetry technique, resolving the compressibility problems, so that the pore size range measured in each case is nearly the same.

On the other hand, we have found no specific surface area values obtained by permeametry in the literature. This technique submits the sample to very similar conditions (percolate by liquid) to that in electrochemical applications as three-dimensional electrode. Besides, we should take into account the thickness ratio. Being this so, we decide to apply the Ergun method to the experimental data found for the carbon felt studied here. The 
previous hydrodinamic study in the literature ${ }^{14}$ confirmed that there is not practically dead zones or wall effect in the system used, at thickness ratio $10 / 8$ to $13 / 8$. This feature permit us to suspect that the results of specific surface area in this same range of thickness ratio are representative for this material.

It is also interesting to remark the consistency between the porosity and specific surface area results obtained from a combination of Ergun method and simulation. The combination of both, the values of porosity and specific surface area obtained by Ergun method, with those from simulation in the equation (10) provides permeability coefficient values very similar to the experimental ones obtained in the present work.

$$
\text { Permeability coefficient }=\frac{\varepsilon^{3}}{\mathrm{~K}^{\prime \prime} \mathrm{A}_{\mathrm{e}}^{2}}
$$

$\mathrm{K}$ " is the Kozeny constant [for media with high porosity $(\varepsilon>0.98)$, it takes a value of $32.8^{24}$ ]

\section{Tortuosity}

The value of tortuosity obtained in this work for the carbon felt is 4.95 , very different than the tortuosity values reported in the literature for $\mathrm{Ni}$ foam (1.15-1.20). As it can be seen, the carbon felt shows values 5-6 times higher 
than the Ni foam but they fall into the common range $2-6^{23}$.

\section{Average pore diameter}

Table 1 shows a summary of the average pore diameters obtained for the carbon felt used in this work and another found in the literature for other carbon felts and three-dimensional electrodes. In some cases, we have calculated the parameter by using the filamentary analog with the dates provided by other authors. Our results obtained from Ergun's method are in agreement with the results calculated by other authors, in spite of the differences in porosity and fibre diameter values. However, the results obtained here from filamentary analog disagree with our other results.

\section{Apparent electrical resistivity}

Table 1 shows a summary of the values for apparent resistivity obtained in this work and other from the literature and, as it can be observed, they are in good agreement for similar materials. And, on the other hand, it is important to remark that the apparent electrical resistivity of carbon felts and, in general, of carbon three-dimensional electrodes, show values 100 times higher than metallic three-dimensional electrodes. This fact is of particular interest because the distribution of the potential inside the threedimensional electrode depends on the electrical conductivity of both the porous medium and the solution ${ }^{28}$. 


\section{Conclusions}

In the present work we have compare a wide variety of experimental techniques, with different theoretical approaches, presenting also unpublished data from the characterization of carbon felts. This permits us to point out that permeametry, with the help of additional information (i.e. RTD Modelling), provides good values for the structural parameters of compressible materials, carbon felts in this case. Therefor, we proposed the combined application of drop pressure measurements and RTD modelling as a new approach to study structural parameters of problematic compressible materials. This technique is particularly suitable for problematic materials such as carbon felt, which presents compressibility and a structural geometry with no simple symmetry (fibres with smooth cylinder like shapes...). The consistency of the interrelation between the values obtained for the different properties reinforces the validity of this method based on the study of fluid flow against other methods based on physical measurements such as the BET approach.

On the other hand, mercury porosimetry indicates that the carbon felt has a bimodal pore size distribution (mesopores and macropores), with a narrow macropore size distribution. And, although some mesoporosity exists in the carbon, its size is relatively small and its amount is not relevant. And also, the $\mathrm{N}_{2} / 77 \mathrm{~K}$ adsorption isotherm indicates the absence of microporosity in 
the carbon felt and a weak adsorbate-adsorbent interaction.

From a structural point of view, the results found in this work, for the characterization of a carbon felt (porosity 0.984, specific surface area 22100-22700 $\mathrm{m}^{2} \mathrm{~m}^{-3}$, tortuosity 5-6, apparent electrical resistivity $2.4 \times 10^{-1}$ - $2.7 \times 10^{-3} \Omega \mathrm{m}$ ), show this is a suitable material to be used as threedimensional electrodes in electrochemical applications.

\section{Acknowledgements}

J. González-García wishes to thank "Consellería de Cultura, Educación y Ciencia" of the Generalidad Valenciana, for his doctoral grant. The authors also thank "D.G.I.C.Y.T." (project QUI97-1086) and "Generalidad Valenciana" again (project GV-2231-94), for their financial support. We also wish to thank Professor J.M. Martín-Martínez for several helpful suggestions.

\section{References}

1 Pletcher and F. C. Walsh, in Industrial Electrochemistry, Chapman and Hall, London 1990, p. 160.

2 F. C. Walsh, in A First Course in Electrochemical Engineering, The Electrochemical Consultancy, Romsey, 1993, p. 142.

3 P. Cognet, J. Berlan, G. Lascoste, P.-L. Fabre, J.-M. Jud, J. Appl. Electrochem., 1995, 25, 1105.

4 J. M. Marracino, F. Coeuret and S. Langlois, Electrochim. Acta, 1987, 32, 1303.

5 S. Langlois and F. Coeuret, J. Appl. Electrochem., 1989, 19, 43.

6 A. Montillet, J. Comiti and J. Legrand, J. Appl. Electrochem., 1993, 23, 1045. 
7 A. Montillet, J. Comiti and J. Legrand, J. Mater. Sci., 1992, 27, 4460.

8 N. Vatistas, P. F. Marconi and M. Bartolozzi, Electrochim. Acta, 1991, 36, 339 .

9 R. Carta, S. Palmas, A. M. Polcaro and G. Tola, J. Appl. Electrochem., 1991, 21, 793.

10 B. Delangue, S. Tellier and M. Astruc, Electrochim. Acta, 1990, 35, 1369.

11 K. Kinoshita and S. C. Leach, J. Electrochem. Soc., 1982, 129, 1993.

12 G. Sánchez-Cano, V. Montiel, V. García, A. Aldaz and E. Elías, in Electrochemical Engineering and Energy, Edited by F. Lapicque et al. Plenum Press, New York 1995, p. 151.

13 D. Pletcher, Watts New, Vol. 2 No. 3, September 1996.

14 J. González-García, J. A. Conesa, J.R. Pérez, G. Codina, V. Montiel and A. Aldaz, Ind. Eng. Chem. Res.(in press).

15 S. Brunauer, P. H. Emmett and T. Teller, J. Am. Chem. Soc., 1938, 60, 309.

16 S. M. Mohnot and E. L. Cussler, Chem. Eng. Sci., 1984, 39, 569.

17 J. Olek, M. D. Cohen and C. Lobo, ACI. Mater J., 1990, 87, 473

18 C. J. Brown, D. Pletcher, F. C. Walsh, J. K. Hammond and D. Robinson, J. Appl. Electrochem., 1994, 24, 95.

19 C. W. Crawford and O. A. Plumb, J. Fluids Eng., 1986, 108, 343.

20 A. Dybbs and R. V. Edwards, in Fundamentals of Transport Phenomena in Porous Media, ed. J. Bear and Y. Corapcioglu, 1984, p. 199.

21 D. Pletcher, I. Whyte, F. C. Walsh and J. P. Millington, J. Appl. Electrochem., 1991, 21, 659.

22 S. Brunauer, L. S. Deming, W. E. Deming and E. Teller, J. Am. Chem. Soc., 1940, 62, 1723.

23 Handbook of Heat and Mass Transfer. Volume 2: Mass Transfer and 
Reactor Design, de. Nicholas P. Cheremisinoff, Gulf Publishing Company, Houston, 1986, p. 439.

24 G. Dogu in Chemical Engineering, Volume 1: Fluid Flow, Heat Transfer and Mass Transfer, ed. J. M. Coulson, J. F. Richardson, J. R. Backhurst and J. H. Harker, Pergamon Press, Oxford, 1978, Chapter 4, p. 125.

25 Y. Oren and A. Soffer, Electrochim. Acta, 1983, 28, 1649.

26 J. Wang, Electrochim. Acta, 1981, 26, 1721.

27 T. L. Hatfield, T. L. Kleven and D. T. Pierce, J. Appl. Electrochem., 1996, 26, 567.

28 F. Coeuret and A. Storck, in Eléments de Génie Electrochimique, TecDoc, Lavoisier, Paris, 1984.

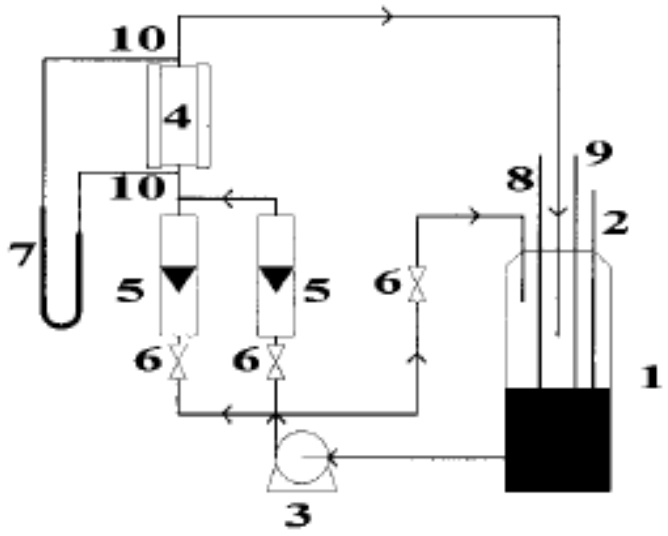

Fig. 1 Diagram of the experimental set-up: 1, reservoir; 2, thermometer; 3, centrifugal pump; 4, test cell; 5, flow meters; 6 , valve; 7 , U-tube manometer; 8 , nitrogen inlet; 9 , heat exchanger; 10 , pressure taps. 
(a)
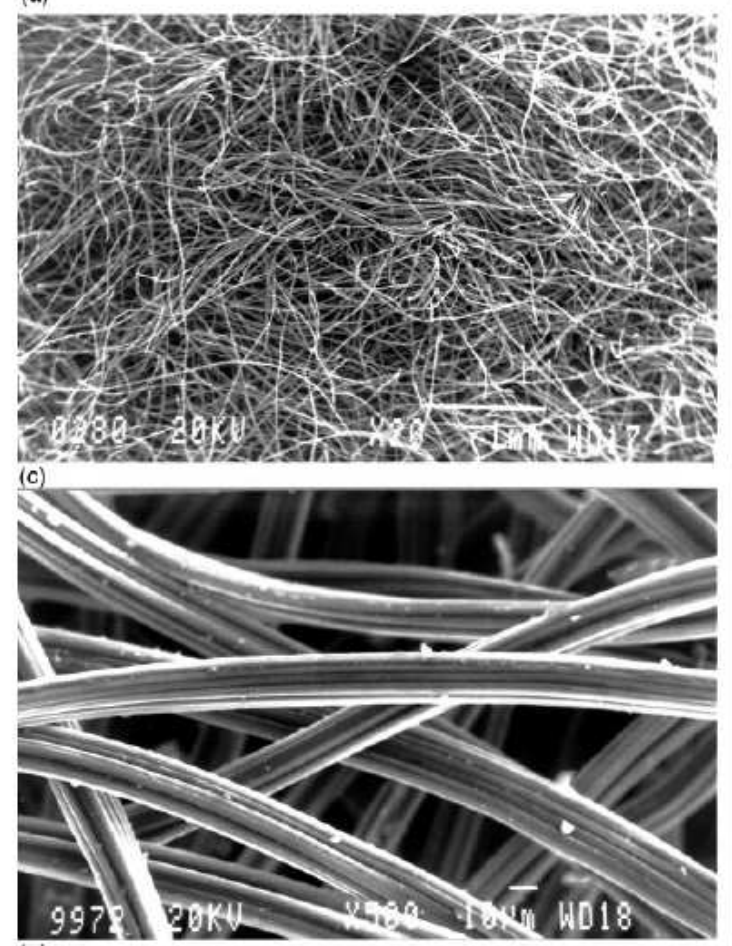

(e)

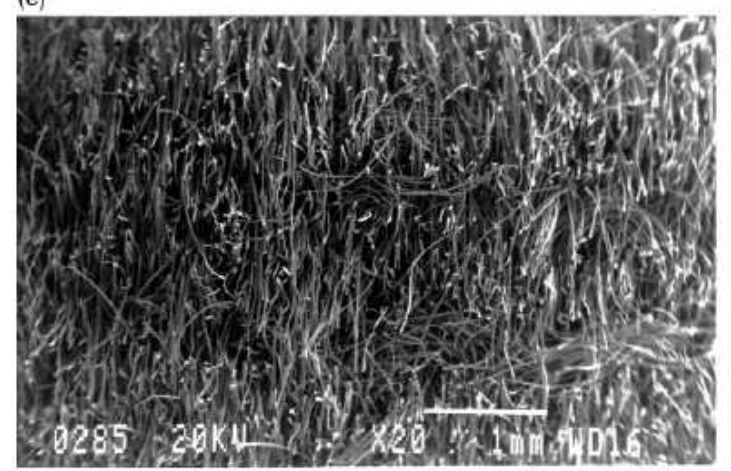

(b)
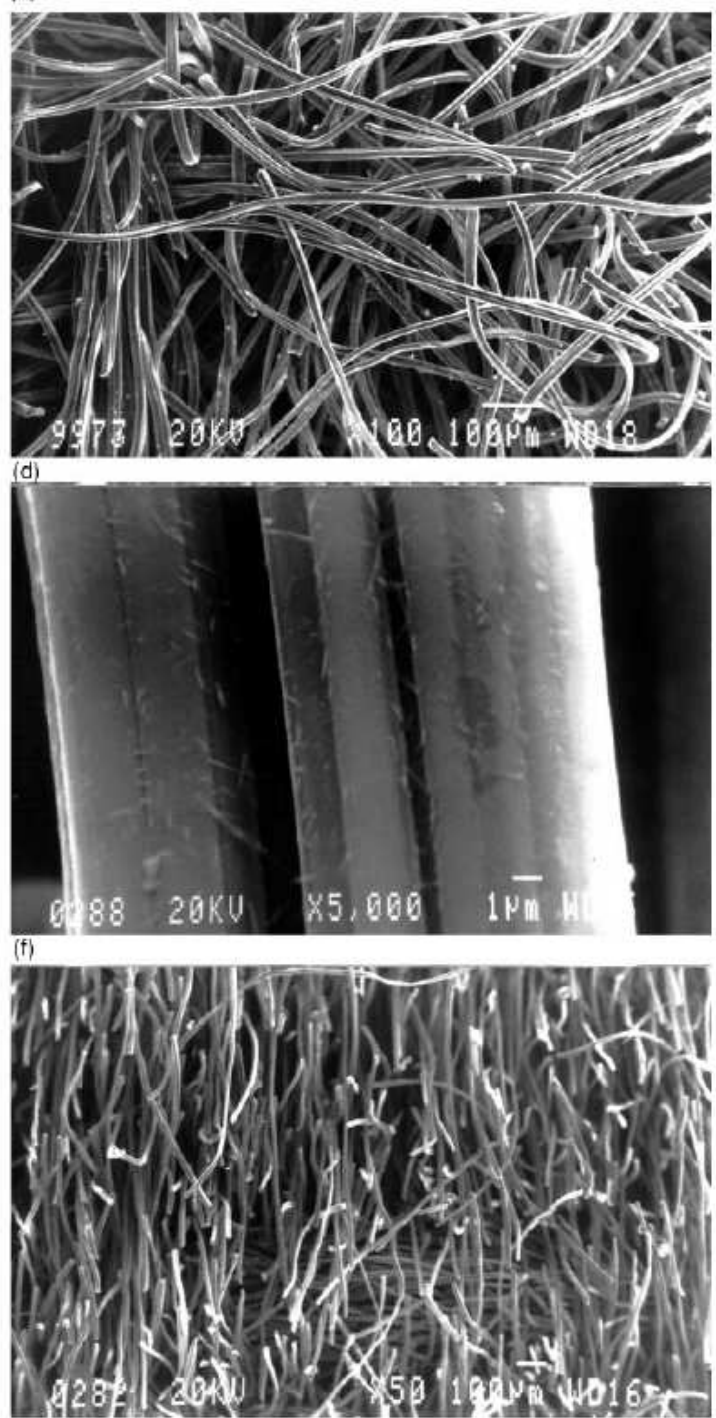

Fig. 2 SEM photographs of the carbon felt taken at various magnifications for the two different sheet orientations. (a) $\times 20,($ b) $\times 100$, (c) $\times 500$, (d) $\times 5000$; sideways (e) $\times 20$, (f) $\times 50$. 


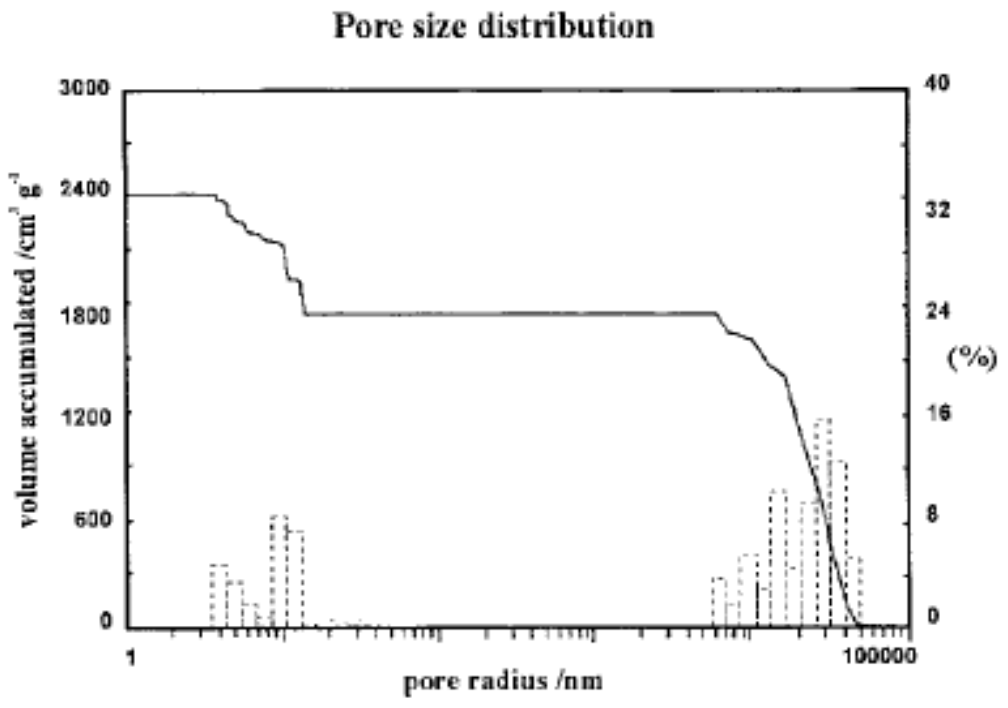

Fig. 3 Cumulative pore volume (left scale) and pore size distribution (right scale) vs. pore radius for RVC 4002 carbon felt electrode from mercury porosimetry.

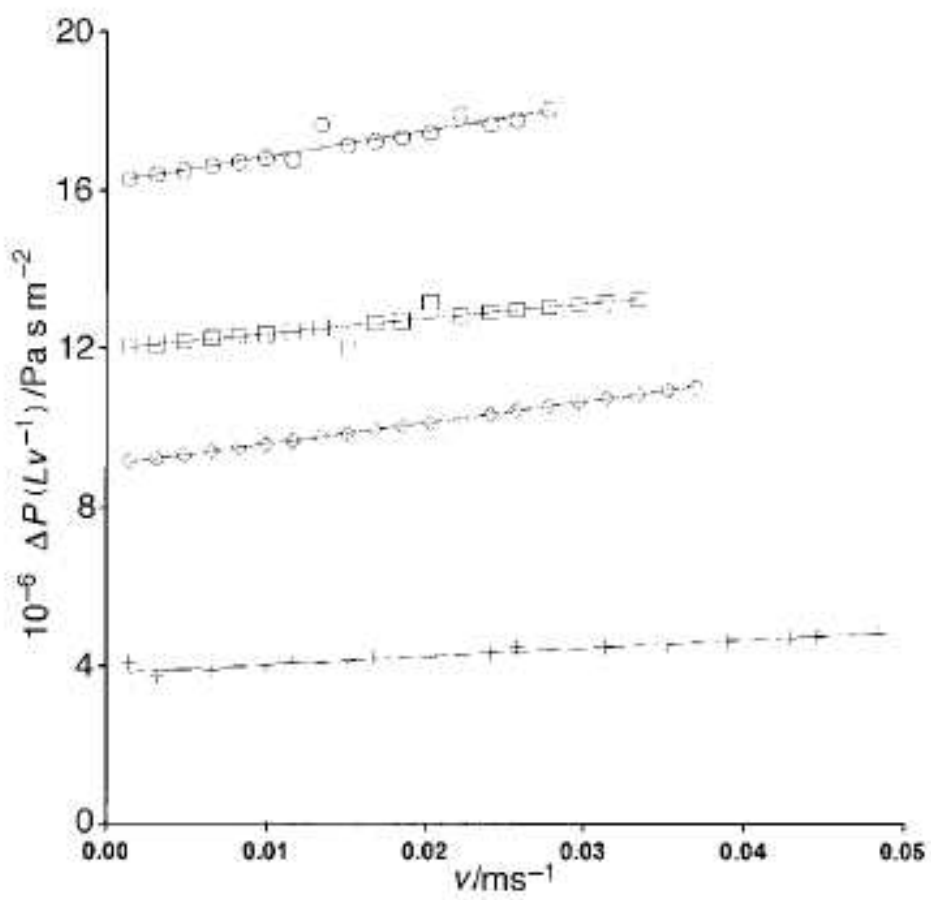

Fig. 4 Ergun plots for the carbon felt/compartment thickness ratios: (+) $8 / 8 ;(\diamond) 9 / 8 ;(\square) 10 / 8 ;(\bigcirc) 13 / 8$. 


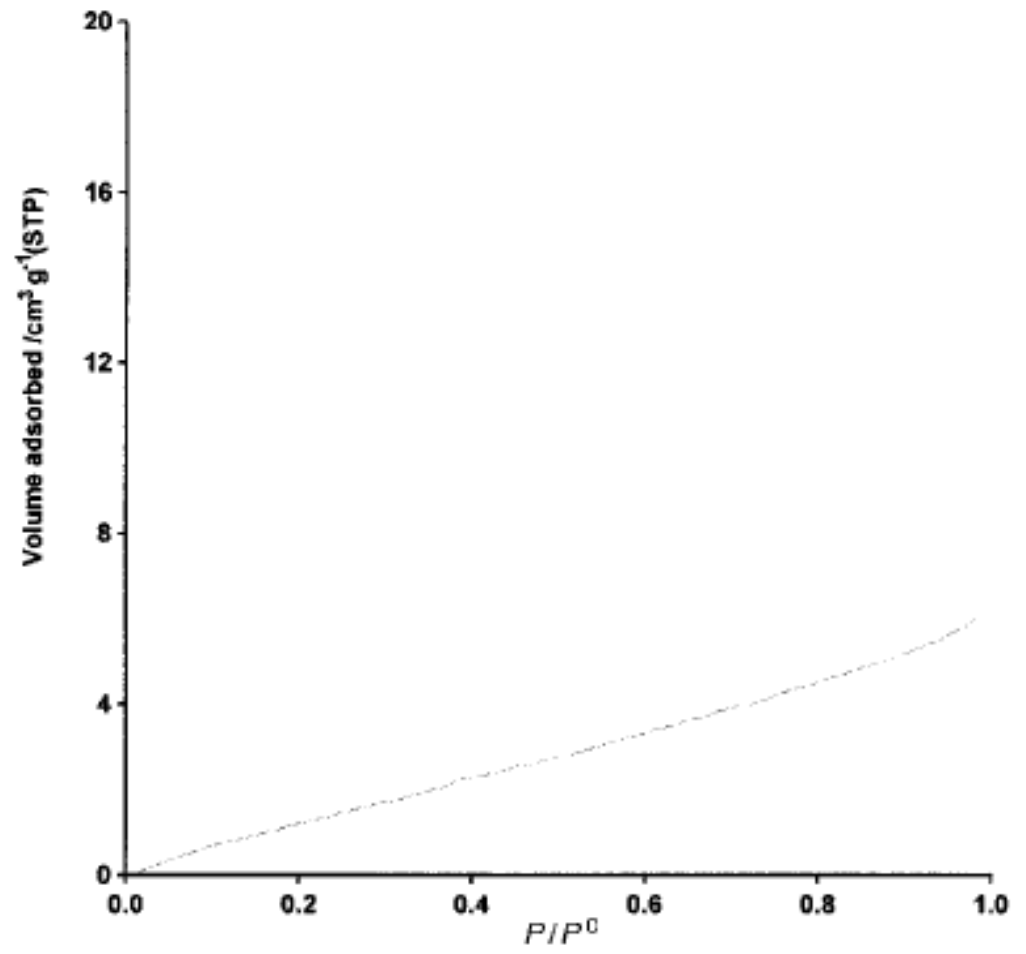

Fig. 5 Adsorption isotherm of $\mathrm{N}_{2}$ at $77 \mathrm{~K}$ on RVC 4002 carbon felt electrode. 


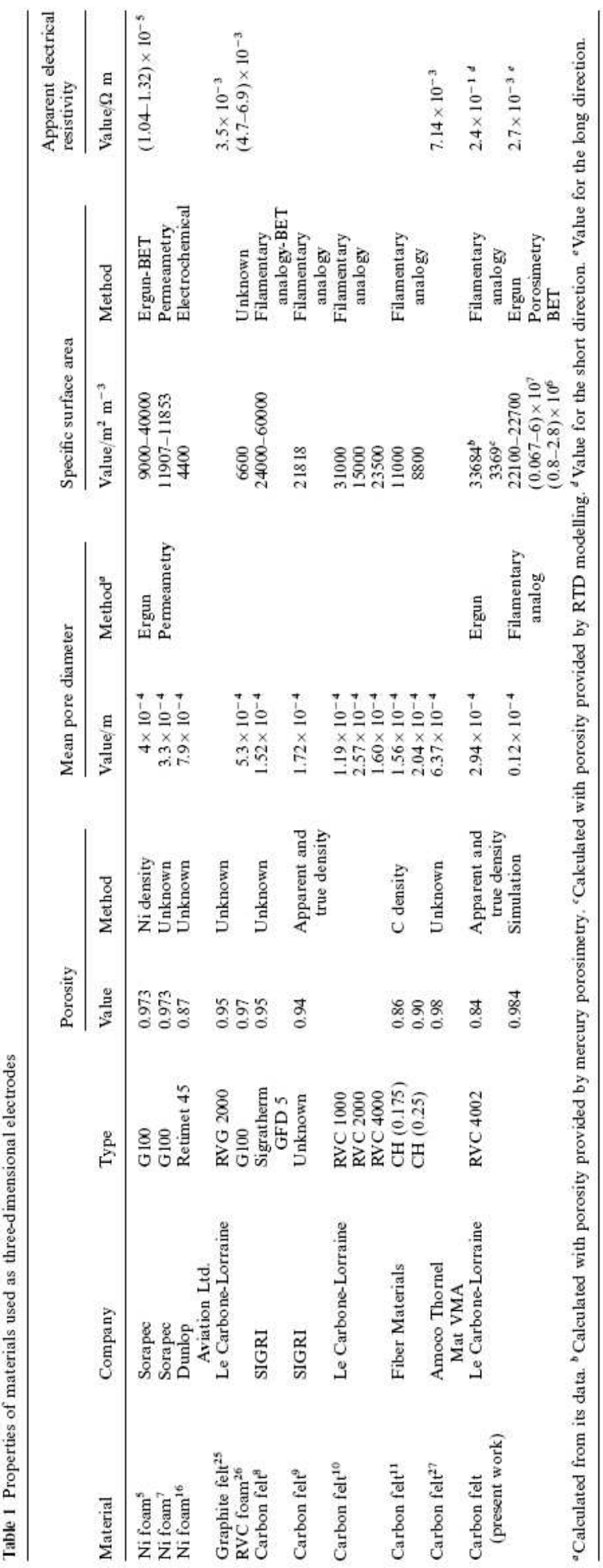

